\title{
To Telescope or Not To Telescope?
}

Timothy F. Slater ${ }^{1,2 *}$

\begin{abstract}
With the rapidly growing number of Internet-accessible and Internet-controlled research-grade telescopes becoming available, astronomy educators are rushing to create authentic researchprograms for students that leverage these new resources. To date, the most successful programs are those that focus on upper-level science-focused students or highly talented high school students with the unifying end-product goal of published research papers. Disciplinebased astronomy education researchers have not yet fully vetted the educational benefits of using these Internet-telescopes, although some early work points toward positive impacts. At the same time, large-enrollment astronomy educators interested in how to scale the use of remotely controlled telescopes and robotically controlled telescopes for large number of non-science majoring students and future teachers are finding few usable classroom-ready solutions. At this point in the evolution of robotic and remote controlled telescopes for formal education, astronomy educators are tacitly assuming that all students should benefit from using telescopes in any form, yet the risk-benefit analysis has not been thoroughly debated within the community to ascertain if the time and energy required for the broader community of all students to use telescopes is worth it.
\end{abstract}

\section{Keywords}

astronomy education research; science teaching; telescopes in education

${ }^{1}$ University of Wyoming

${ }^{2}$ CAPER Center for Astronomy \& Physics Education Research

${ }^{*}$ Corresponding author: timslaterwyo@gmail.com

\section{Introduction}

Unquestionably, the single scientific instrument that comes to mind when most people think about astronomy is the telescope. Most scientists - and some members of the general public — can enthusiastically relate a story of the first time they looked through a telescope. As a natural consequence, astronomy educators have long yearned for ways to connect telescopes with students to create authentic learning experiences that mimic the experience of doing astronomy.

Educators have previously documented the inherent difficulties in providing telescope-based learning experiences for students, particularly at large enrollment scales (viz., for example, Slater et al., 2014). These barriers to using telescopes for teaching include: the vast majority of people live in light-polluted cities making observations difficult; formal schooling is largely a daytime activity whereas telescope-astronomy is largely a nighttime activity; the immense cost of acquiring telescopes on the part of schools; and the lack of many science educators having substantive expertise in using telescopes, to name but a few challenges. These challenges seem to be long-standing and difficult to mitigate easily.

Upon the advent of using the Internet for accessing, controlling, and programming high quality scientific instruments, astronomy educators have enthusiastically hoped for the availability of low-cost, no-cost Internet-controlled telescopes for 
their students (Gershun et al., 2014; Gershun et al., 2012; Gomez and Fitzgerald, 2017; Slater, 2007).

Yet, as astronomy educators enter the fourth decade of widespread Internet access, the hope and promise of a future with a telescope-observing experience for every student edges ever closer, but still seemingly just out of reach, particularly for those who work with non-science majoring undergraduates and high school teachers managing large enrollments.

Given that there are still a few moments before telescope-learning experiences do finally avail themselves to nearly every astronomy student, it seems reasonable, if not prudent, to consider whether using telescopes as an educational experience is worth doing, given the time and energy necessary to include telescope learning activities in the often already overburdened curriculum most students are subjected to. Even if questioning the tacit assumption that students should use telescopes seems to be merely an academic exercise, conscientious educators need be able to justify theoretically and experimentally what students could and should be learning with a telescope, why using telescopes this is more valuable than the opportunity cost of other academic experiences, and how the learning will be assessed BEFORE engaging in expending considerable energy developing the effective learning experiences and learning activities themselves (Brissenden et al., 2001). In other words, following the famous quotation, "To be, or not to be" from the opening phrase of a soliloquy spoken by Prince Hamlet in the so-called "nunnery scene" of William Shakespeare's play Hamlet, I propose that the questions astronomy educators need to be able to answer is "To Telescope, or not to Telescope, that is the question."

\section{What Can Astronomical Science Look Like?}

Professors, politicians, philosophers, teachers, and policy makers, among many others, have long debated what science looks like, and what science should look like in schools. For example, ongoing questions of should students be learning about the results and influence of science or, in contrast, should students be doing science in the course of their schooling? A cursory survey of most early years undergraduate courses in physics and astronomy looks like the student experience is largely solving end-of-chapter homework problems rather than mimicking the daily work of contemporary scientists. And, when considering what is required for students to "do science", there is simply insufficient time and resources-and perhaps societal interest in-being sure that all students participate in both types of learning.

In an effort to move beyond seemingly endless debate to something actionable, I recommend our community considers what the National Research Council (2007) says about students who are proficient at science as being a baseline guide for considering how telescopes in education can or cannot adequately contribute to the national interest in improving science education. The NRC book Taking Science to School (2007), states that four basic and foundational characteristics of students who are proficient in science are those who demonstrably:

1. Know, use and interpret scientific explanations of the natural world.

2. Generate and evaluate scientific evidence and explanations.

3. Understand the nature and development of scientific knowledge.

4. Participate productively in scientific practices and scientific discourse.

If these four characteristics are able guide our thinking about what using telescopes in education might look like, then I argue that our community of educators can make real progress. Alternatively, if our community goals are simply to make telescopes accessible using a "build it and they will come" attitude, then I worry that our efforts will be largely wasted. The bottom line here is that to 
meaningfully answer the question of "to telescope or not to telescope?", one might be wise to consider if using telescopes as an educational experience moves us closer to achieving the four points listed above.

\section{First Steps To Success}

With the rapidly growing number of Internet-accessible and Internet-controlled research-grade telescopes becoming available, astronomy educators are rushing to create authentic research-programs for students that leverage these new resources. Unquestionably, there have been some tremendously successful programs, built upon a foundation of incredibly enthusiastic, tireless, and knowledgably individuals, yet these are too few and far between to impact large numbers of students. To date, the most successful of these programs are those that focus on very small numbers of science-focused students or highly talented high school students with the unifying end-product goal of published research papers (viz., for example, Proceedings of 2017 Robotic Telescopes, Student Research \& Education Conference).

There are even far fewer efforts attempting to engage large numbers of students. Astronomy educators serving large-enrollment settings who are interested in how to scale the use of remotely controlled telescopes and robotically controlled telescopes for large numbers of non-science majoring students and future teachers are finding essentially no classroom-ready solutions. There are a few promising avenues: One of the most notable is the MicroObservatory project (Gould et al., 2006). But, to be distantly objective, at this point in its evolution, most astronomy educators are tacitly assuming that all students would benefit from using telescopes in any form, yet the risk-benefit analysis has not been thoroughly debated within the community to ascertain if the time and energy required for students to use telescopes is worth it. It is easy to forget that every new educational activity included in the astronomy learning sequence means that something else should be dropped-an educational opportunity cost of sorts.

\section{Opportunities for Astronomy Education Research}

While research and development in remote and robotically controlled telescopes is ongoing, so is the field of discipline-based astronomy education research (Slater et al., 2015). The hope is that astronomy education researchers can provide guidance and insight into how to best improve student learning by focusing on understanding the underlying mental mechanisms and motivational aspects of using telescopes. Unfortunately, discipline-based astronomy education researchers have not yet fully vetted the educational benefits of using these Internet-telescopes, although some early work points toward positive impacts while others point to ponderous results.

One of the ponderous works worth considering in the present context is that by Berryhill and Slater (2013). Educators have long participated in informal water-cooler debates about if students would benefit and enjoy astronomy more if they were using their own, hard-earned images or using professional images acquired from data-mining. In surveying college students about whether they would rather use professional astronomy images or images that they've taken themselves, results were mixed depending on precisely how one asks the question. Based on a lens of ownership, one might assume that students would like their own hard-earned image better. Based on a lens of usefulness, one might assume that professional astronomy images might be preferred. The simple testing of which is preferred was not so straightforward. Compare the differing results of Figure 1 and Figure 2.

We interpret this data as students being highly utilitarian. In other words, it is less important that telescope educators focus on whether or not students like their pictures better or worse than those of professional astronomers, and more important to figure out what we want students to experience. Such a perspective leads one to propose what might work best is a series of 


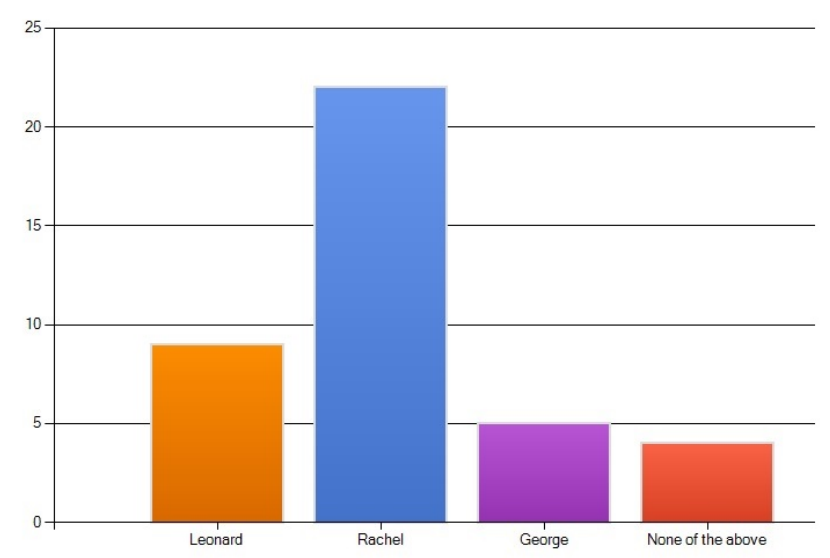

Figure 1. With which classmate do you most agree? (i) Leonard: I like the image I made the best because I took it; (ii) Rachel: I like the image from the professional telescope best because it's crisp and clear. What do you think, George? (iii) George: I don't know. Adapted from Berryhill et al. (2013))

educational experiences a students should have on the road to using a robotically controlled telescope, if the educational objectives of the NRC (2007) listed above are to be met.

\section{A Proposed Learning Progression}

In brief, my colleagues and I propose that most students are generally unable to simply start using a robotically controlled, batch queue telescope and fully conceptualize the event. My analysis and experience together suggest that students would benefit from first using a eyepiece based telescope, then a live-remotely controlled telescope, before being able to benefit from the use of a robotically controlled telescope. In support of this idea, we proposed in 2014 and have refined since then the following learning progression for telescopes in education (adapted from Slater et al. (2014)):

1. Small dots of light in the night sky, called stars, are visible each clear night

2. Stars have names and positions in the sky that can be described and located on sky maps

3. Stars change their position in the sky over the course of a night and over the year

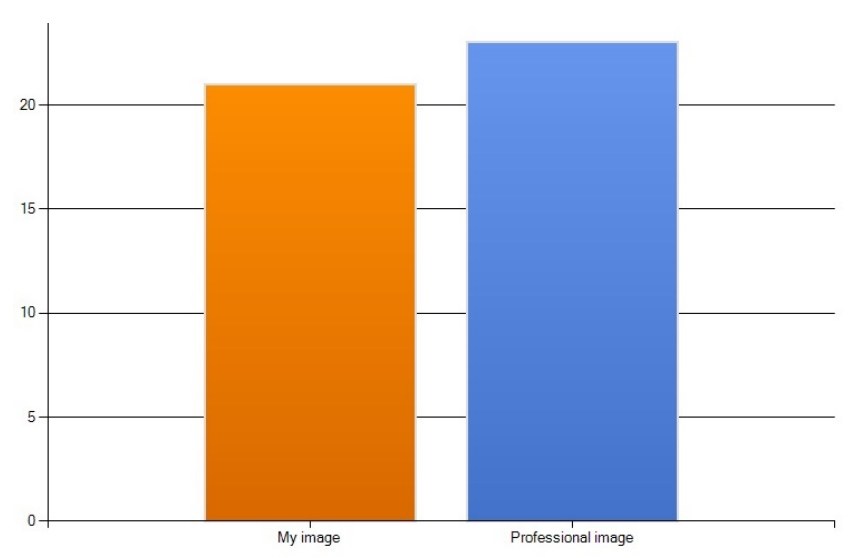

Figure 2. If you were going to show a friend or a family member a picture of Saturn, which would you use? (adapted from Berryhill et al. (2013))

4. Most stars look like infinitely small dots through a telescope pointed at a prescribed location

5. Some star-like objects are not stars, but other objects like planets, and appear different in a telescope

6. Some nearby objects are visible in a telescope are not visible to the naked eye, such as Jupiter's moons, Saturn's rings, craters on the moon, phases of Venus

7. Some distant objects visible in a telescope are not visible to the naked eye, such as galaxies, nebulae, and even other stars 8 . Data from a telescope can be gathered through hand-drawn sketches, digital photographs, and other less familiar scientific instruments and take the form of an image or set of numbers

8. Color-images are created by combining and overlaying multiple images in different wavelengths

9. A computer can be programmed to point a telescope and gather data for the observer in real-time at nearby or faraway locations gather data for the observer in real-time at nearby or faraway locations 
10. A computer can be programmed to point a telescope and gather data for the observer at a faraway location, including in outer space, even when the observer is not nearby or even awake

11. A computer can be programmed to point a telescope and gather data for the observer at a faraway location, including in outer space, even when the observer is not nearby or even awakelocation, including in outer space, even when the observer is not nearby or even awake

12. Computer-controlled telescopes fill online databases that can be accessed over the Internet for analysis

13. Detailed analysis of astronomical objects can be accomplished by assigning a data collection task to a robotically controlled telescope or by querying a database of previous observations

\section{Discussion}

Taken together, if students are to participate in scientific practices and scientific discussion, then a specific sequence of education experiences are likely required. I propose that there is a minimum of four sequential steps than cannot be skipped for the majority of students. These are, in order:

1. Look through a telescope, then

2. operate a telescope by hand, then

3. remotely control a telescope in real-time, then, finally, students will be ready to appreciate, value, and comprehend the task of,

4. submit an online request for a robotically-controlled telescope to collect desired data (or data mine)

My observation is that well intentioned professional astronomers all too often mistakenly assume that students can immediately connect the notion that a real-time, locally controlled telescope and a distant, batch-queue controlled telescope do the same job. For most novices, these two seemingly similar entities are in different universes, and require more than a single "night" experience to be effective.

Following this proposed learning sequence has an additional benefit. Those readers who have had the opportunity to serve as judges at middle school science fairs know all to well that many science fair project evaluation checklists require students to actively collect their own data. In far too many cases, good astronomy science fair projects based on data mining or online datasets fail to have a chance at winning because too many people - including active scientists - believe that authentic science requires one to collect their own data. As a result, projects such as data mining or robotically controlled telescopes fail to meet their mistaken litmus test for authentic science. Being sure students have gone through each these steps can help to mitigate this widespread misconception about how contemporary science is done.

The educational approach I am advocating here is that if one decides that it is worth the educational time, expense, and opportunity cost to include telescopes in education, then such an effort should not be done quickly over a single night; but, instead, requires a conscious commitment of time and energy that probably works best when it includes an evolving sequence of scaffolded learning experiences that are far more than simply assigning students the complex task of telling a computer to go make some astronomical observations. In other words, if you are going "to telescope" then be sure to fully commit your scarce time allocations and "to telescope" extensively because simply giving students a brief experience is probably insufficient to have longstanding, durable educational benefits.

\section{Acknowledgements}

This work has been supported in part by the Wyoming Excellence in Higher Education Endowment and the CAPER Center for Astronomy 
\& Physics Education Research. The author wishes to acknowledge the insights and contributions of Dr. Katie J. Berryhill and Dr. Stephanie J. Slater in developing this theoretical work.

\section{References}

Berryhill, K., Coble, K., Slater, T., McLin, K., and Cominsky, L. (2013). Using Internet-Based Robotic Telescopes to Engage Non-Science Majors in Astronomical Observation. In AGU Fall Meeting Abstracts.

Brissenden, G., Slater, T. F., Mathieu, R. D., et al. (2001). The role of assessment in the development of the college introductory astronomy course. Astronomy Education Review, 1(1):1-24.

Council, N. R. et al. (2007). Taking science to school: Learning and teaching science in grades K-8. National Academies Press.

Gershun, D., Berryhill, K., and Slater, T. (2012). Evaluating SLOOH robotic telescopes for formal educational use. In AGU Fall Meeting Abstracts.

Gershun, D. C., Slater, T. F., and Berryhill, K. J. (2014). Mixed-Methods Study that Examines Nine Science Teachers' Perceptions of SLOOH Robotic Telescope for Teaching Astronomy. Latin American Journal of Astronomy Education (RELEA), No. 17, p. 7-37, 17:7-37.

Gomez, E. L. and Fitzgerald, M. T. (2017). Robotic telescopes in education. Astronomical Review, 13(1):28-68.

Gould, R., Dussault, M., and Sadler, P. (2006). What's Educational about Online Telescopes?: Evaluating 10 Years of MicroObservatory. Astronomy Education Review, 5(2).

Slater, S. J., Slater, T. F., Heyer, I., and Bailey, J. M. (2015). Conducting Astronomy Education Research: An Astronomer's Guide, 2nd Edition. Pono Publishing.

Slater, T. (2007). Using remote-control telescopes in ASTR 101. In Cosmos in the classroom symposium on teaching introductory astronomy. San,
Francisco, CA: Astronomical Society of the Pacific.

Slater, T. F., Burrows, A. C., French, D. A., Sanchez, R. A., and Tatge, C. B. (2014). A proposed astronomy learning progression for remote telescope observation. Journal of College Teaching \& Learning (Online), 11(4):197. 